\title{
Vaccination Knowledge and Perception among Pharmacy Undergraduates in a Northern Public University in Malaysia
}

\author{
Tinagaran A/L Karunakaran, Balamurugan A/L Tangiisuran, Nur Hafzan Md \\ Hanafiah. \\ School of Pharmaceutical Sciences, Universiti Sains Malaysia, Penang \\ DOI: http://dx.doi.org/10.31344/ijhhs.v5i0.319
}

Introduction: Worldwide, pharmacists are one of the advocates and practitioners which are commonly overlooked in improving vaccine hesitancy.

Objectives: The aim of this study is to explore the vaccination knowledge and perception level as well as the sources of information sought, to support the importance in including the topic within the existing pharmacy curriculum.

Methods: A cross sectional study using a validated and tested 43-itemed questionnaire were conducted among pharmacy students in USM. It included 4 domains of demographic data, knowledge on vaccination, perception towards vaccination and source of information on vaccination. Scoring and grouping of knowledge and perception level is based on modified Bloom's cut-off point. A confidence interval of $95 \%$, $p$ value $\leq 0.05$. Chi-square, ANOVA and the Kruskal-Wallis test were considered for the analysis of data.

Results: Among 478 eligible pharmacy undergraduates, $311(65 \%)$ participated and completed the questionnaire with a majority of female's respondents $(76.5 \%)$. Nearly $72.1 \%$ of the students had fair level of knowledge and $90 \%$ had good perception level towards vaccination. Online resource shown to be the favourable source of information onvaccination $(84.2 \%)$. Furthermore, there was a statistically significant knowledge difference across the age, study year and races, $(p=.02),(p<0.05)$ and $(p<0.05)$ respectively. On the other hand, the level of perception shown to have a significant association with nationality $(\mathrm{p}=0.016)$, gender $(\mathrm{p}=0.049)$ and $\operatorname{race}(\mathrm{p}=0.002)$.

Conclusion: Majority of the students were shown to have fair knowledge and good perception towards vaccination, suggesting the need of implementing vaccination content delivery in the existing curricula to ensure better understanding and practice towards vaccination advocacy.

Keywords: vaccination, pharmacy undergraduates, knowledge, perception, questionnaire 